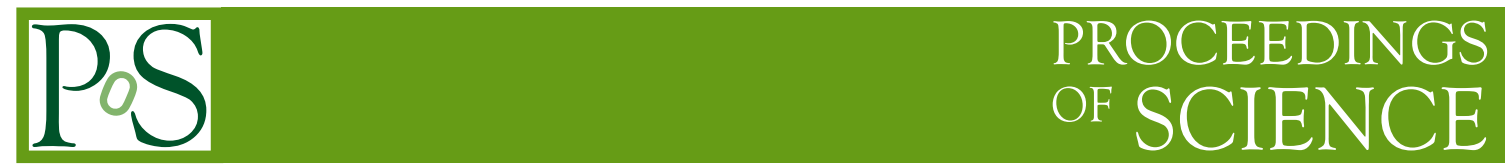

\title{
Status of chiral perturbation theory
}

\author{
Gerhard Ecker ${ }^{* \dagger}$ \\ Faculty of Physics, University of Vienna, Boltzmanngasse 5, A-1090 Wien \\ E-mail: gerhard.ecker@univie.ac.at
}

The status of chiral perturbation theory in the meson sector is reviewed. The main emphasis is on recent developments in pion pion scattering, in semileptonic decays and in nonleptonic kaon decays. A few other selected topics are also discussed.

8th Conference Quark Confinement and the Hadron Spectrum

September 1-6, 2008

Mainz, Germany

\footnotetext{
* Speaker.

${ }^{\dagger}$ Supported in part by EU contract No. MRTN-CT-2006-035482 (FLAVIAnet)
} 


\section{Introduction and overview}

Chiral perturbation theory (CHPT) was introduced nearly 30 years ago [1]. Soon thereafter, it was developed [2, 3] into a powerful tool for treating the strong interaction at low energies $(E \ll 1$ $\mathrm{GeV}$ ). From the first steps beyond current algebra, CHPT has come a long way as manifested by the present-day chiral Lagrangian in the meson sector ${ }^{1}$ displayed in Table 1. In addition to the strong interactions of mesons, the Lagrangian also accounts for nonleptonic weak interactions and it includes the photon and leptons as dynamical degrees of freedom. CHPT aims at reliable results in the confinement regime to allow for conclusive tests of the Standard Model and to search at the same time for traces of new physics at low energies.

\begin{tabular}{|c|c|}
\hline \multicolumn{1}{|c|}{$\mathscr{L}_{\text {chiral order }}$ \# of LECs $)$} & loop order \\
\hline $\mathscr{L}_{p^{2}}(2)+\mathscr{L}_{p^{4}}^{\text {odd }}(0)+\mathscr{L}_{G_{F} p^{2}}^{\Delta S=1}(2)+\mathscr{L}_{G_{8} e^{2} p^{0}}^{\text {emeak }}(1)$ & $L=0$ \\
$+\mathscr{L}_{e^{2} p^{0}}^{\text {em }}(1)+\mathscr{L}_{\text {kin }}^{\text {leptons }}(0)$ & \\
$+\mathscr{L}_{p^{4}}(10)+\mathscr{L}_{p^{6}}^{\text {odd }}(23)+\mathscr{L}_{G_{8} p^{4}}^{\Delta S S=1}(22)+\mathscr{L}_{G_{27} p^{4}}^{\Delta S=1}(28)$ & $L \leq 1$ \\
$+\mathscr{L}_{G_{8} e^{2} p^{2}}^{\text {emeak }}(14)+\mathscr{L}_{e^{2} p^{2}}^{\text {em }}(13)+\mathscr{L}_{e^{2} p^{2}}^{\text {leptons }}(5)$ & \\
$+\mathscr{L}_{p^{6}}(90)$ & $L \leq 2$ \\
\hline
\end{tabular}

Table 1: The mesonic Lagrangian for chiral $S U(3)$ in use today, including strong, electromagnetic and nonleptonic weak interactions. The leptons must be incorporated for radiative corrections in semileptonic decays. The numbers in brackets denote the number of low-energy constants (LECs).

As Table 1 shows, higher orders in the chiral expansion are accompanied by an increasing number of LECs. The determination of those LECs has been and will continue to be essential for progress in the field. The following methods are being employed.

i. The LECs can be determined by confronting CHPT predictions with experimental data. This straightforward approach encounters its limits already at NLO for nonleptonic weak decays and at NNLO for strong processes: there are too many LECs for the experimental information available to obtain a predictive scheme.

ii. In some cases, combining chiral amplitudes with dispersion theory has proven to be fruitful.

iii. Lattice methods have made tremendous progress [5, 6].

iv. Large- $N_{c}$ motivated resonance saturation has provided a number of successful estimates [7].

The purpose of this talk was to discuss some major achievements of CHPT in the meson sector during the past two years: pion pion scattering, semileptonic decays, nonleptonic $K$ decays and a few other selected topics.

\footnotetext{
${ }^{1}$ The baryon sector is covered by Meißner [4].
} 


\section{Pion pion scattering}

Pion pion scattering at low energies is a fundamental process for chiral $S U(2)$. It is in particular very sensitive to the mechanism of spontaneous chiral symmetry breaking. The ultimate theoretical result was obtained by combining dispersion theory [8] with CHPT. The dispersive amplitude depends on experimental data for $E_{\pi \pi} \geq 800 \mathrm{MeV}$ and on two subtraction constants. By matching the dispersive amplitude with the chiral amplitude to NNLO [9], the two subtraction constants can be expressed in terms of the S-wave scattering lengths [10]

$$
a_{0}^{0}=0.220 \pm 0.005, \quad a_{0}^{2}=-0.0444 \pm 0.0010 .
$$

When comparing theoretical predictions with experimental results, one must keep in mind that the chiral amplitudes and the scattering lengths (2.1) refer to an isospin symmetric world. Isospin violation and radiative corrections must therefore be taken into account before making the comparison. Experimental information comes from three sources.

a. $K_{e 4}$ decays;

b. Decay of pionium;

c. Cusp in $K \rightarrow 3 \pi$ decays.

I will restrict the discussion to items a) and c) where important developments, both in experiment and in theory, have occurred during the last two years.

\section{$2.1 K^{+} \rightarrow \pi^{+} \pi^{-} e^{+} v_{e}$ decays}

$K_{e 4}$ decays are the traditional source for accessing pion pion scattering at low energies. From the final state interaction of the two pions one can extract the phase shift difference $\delta_{0}-\delta_{1}$ where $\delta_{0}, \delta_{1}$ are the phase shifts for the $I=0 \mathrm{~S}$-wave and the $I=1 \mathrm{P}$-wave, respectively, in the isospin limit and in the absence of electromagnetic corrections.

In addition to the radiative corrections applied by the experimental groups, isospin violation due to the pion mass difference and to $m_{u}-m_{d}$ has turned out to be important. The most recent analysis at the one-loop level is described in Ref. [11] where also references to related previous and ongoing work can be found. In the one-loop diagrams for $K_{e 4}$, the physical $\pi^{+}, \pi^{0}$ masses must be inserted and an additional diagram involving $\pi^{0}-\eta$ mixing appears. Denoting the experimentally accessible phase shifts by $\psi_{0}, \psi_{1}$, the authors of Ref. [11] obtain for the measurable S-wave phase shift $\psi_{0}(s)$ in the elastic region $4 M_{\pi^{+}}^{2}<s<16 M_{\pi^{0}}^{2}\left(\psi_{1}=\delta_{1}\right.$ to the order considered)

$$
\psi_{0}(s)=\frac{1}{32 \pi F^{2}}\left\{\left(s+4 M_{\pi^{+}}^{2}-4 M_{\pi^{0}}^{2}\right) \sigma\left(M_{\pi^{+}}\right)+\left(s-M_{\pi^{0}}^{2}\right)\left(1+\frac{3\left(m_{d}-m_{u}\right)}{2\left(m_{s}-\hat{m}\right)}\right) \sigma\left(M_{\pi^{0}}\right)\right\}+O\left(p^{4}\right)
$$

where

$$
\sigma(M)=\sqrt{1-4 M^{2} / s}, \quad \hat{m}=\frac{1}{2}\left(m_{u}+m_{d}\right) .
$$

The difference $\psi_{0}-\delta_{0}$ to be subtracted from the measured phase shift $\psi_{0}(s)$ is shown in Fig. 1 [11]. The isospin corrected scattering lengths from the NA48 experiment at CERN [12] now agree 
perfectly with the theoretical prediction [10] (the small red ellipse in Fig. 1) whereas the agreement is now less impressive for the BNL experiment E865 [13]. For further details I refer to the contribution of Bloch-Devaux in these Proceedings [14].
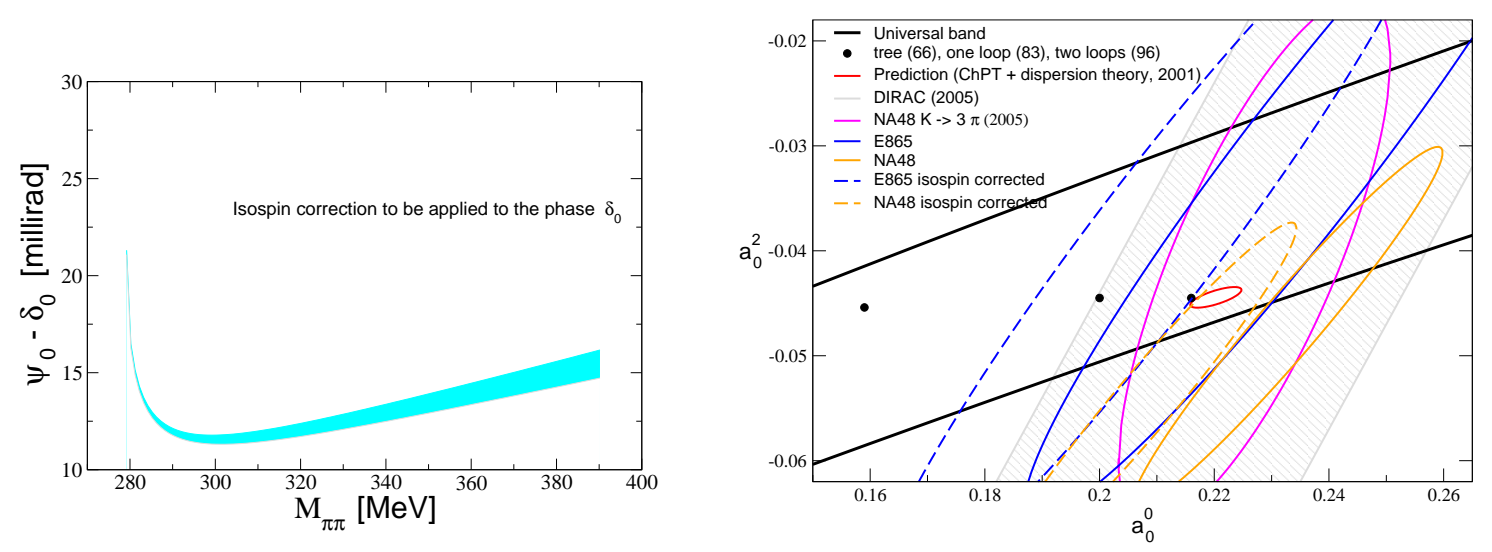

Figure 1: Isospin violating corrections to be applied to the measured S-wave phase shift (left figure). Comparison between theory and experiment for the scattering lengths (right figure). Both figures are taken from Ref. [11].

\subsection{Cusp in $K \rightarrow 3 \pi$ decays}

The cusp was first seen in the $M_{\pi^{0}} \pi^{0}$ distribution in $K^{ \pm} \rightarrow \pi^{ \pm} \pi^{0} \pi^{0}$ [15], more recently also in $K_{L} \rightarrow 3 \pi^{0}$. It is due to the rescattering of pions in the final state [16, 17]

$$
K^{ \pm} \rightarrow \pi^{ \pm}\left(\pi^{+} \pi^{-}\right)^{*} \rightarrow \pi^{ \pm} \pi^{0} \pi^{0} .
$$

The basic mechanism is an interference between tree and 1-loop amplitudes. The square-root singularity generates a cusp above threshold at $M_{\pi^{0} \pi^{0}}^{2}=4 M_{\pi^{+}}^{2}$. From (2.4) the effect is seen to be sensitive to the combination of $\pi \pi$ scattering lengths

$$
a_{0}^{0}-a_{0}^{2} \sim A\left(\pi^{+} \pi^{-} \rightarrow \pi^{0} \pi^{0}\right)_{\text {thresh }} .
$$

Various approaches have been pursued to extract $a_{0}^{0}-a_{0}^{2}$ from $K \rightarrow 3 \pi$ near threshold.

i. Following the original approach of Cabibbo [16] based on unitarity and analyticity, a systematic expansion of the singular terms of the $M_{\pi^{0} \pi^{0}}$ distribution in powers of the scattering lengths was performed in Ref. [18].

ii. In a related method, unitarity and analyticity were combined with CHPT [19].

iii. A two-loop dispersive representation of $K \rightarrow 3 \pi$ amplitudes in the presence of isospin breaking is under construction [20].

iv. In the most advanced approach based on a nonrelativistic effective field theory, the $K \rightarrow 3 \pi$ amplitudes are expanded in powers of the scattering lengths and of the pion momenta in the $K$ rest frame [21]. Most recently, radiative corrections have been performed within this framework [22]. In contrast to standard CHPT, this approach is valid to all orders in the quark masses. 
An up-to-date comparison between theory and experiment can be found in the contribution of Bloch-Devaux [14].

\section{Semileptonic decays}

Semileptonic decays have long been a rich field for CHPT [23].

\section{1 $\mathrm{K}_{13}$ decays}

$K_{l 3}$ decays are at present still the best source for the CKM matrix element $V_{u s}$. They have therefore been investigated intensively during recent years [24]. A possible problem with the slope of the scalar form factor $f_{0}(t)$ was discussed by Leutwyler [6]. For the experimental analysis, the complete radiative corrections in a CHPT framework are now available for both $K_{e 3}$ and $K_{\mu 3}$ [25]. The collaboration between theory and experiment has led to a very precise value [24] for the product

$$
\left|V_{u s}\right| f_{+}^{K^{0} \pi^{+}}(0)=0.21661(47) \text {. }
$$

The predictions for the vector form factor at $t=0$ show a certain spread, but are dominated now by the lattice results (see Fig. Q). With $f_{+}^{K^{0} \pi^{+}}(0)=$ 0.964(5) [26] one obtains $\left|V_{u s}\right|=0.2246(12)$, in perfect agreement with CKM unitarity, taking $\left|V_{u d}\right|$ from nuclear $\beta$ decay.

Figure 2: Various predictions for $f_{+}^{K^{0}} \pi^{+}(0)$ collected in Ref. [24].

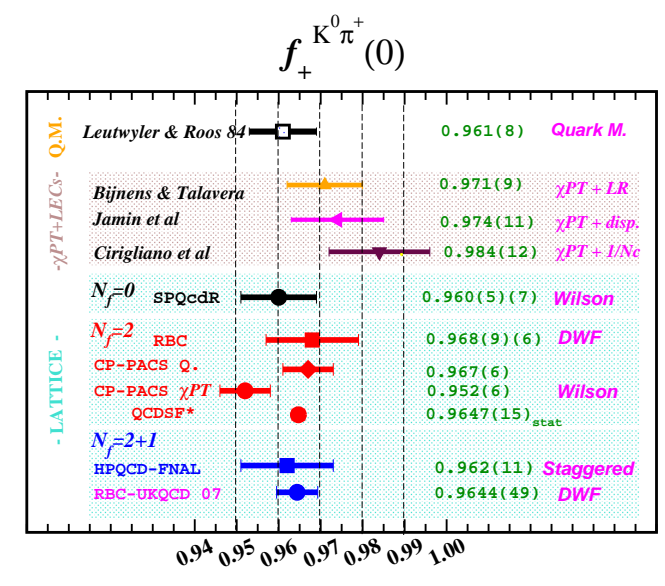

For the sake of playing the devil's advocate, two caveats are worth mentioning.

- Hadronic $\tau$ decays are becoming competitive for the determination of $\left|V_{u s}\right|$. Taking into account (small) $S U$ (3) breaking effects, Gamiz et al. obtained [27]

$$
\left|V_{u s}\right|=0.2165(26)_{\text {expt }}(5)_{\text {theor }}
$$

in agreement with a similar more recent analysis of Maltman et al. [28]. The full data samples from BELLE and BaBar are needed for a definitive conclusion.

- The most recent value $\left|V_{u d}\right|=0.97408$ (26) from superallowed $0^{+} \rightarrow 0^{+}$nuclear $\beta$ decay [29] has an uncertainty that is only half the theoretical uncertainty in pionic $\beta$ decay [30], a much simpler process from the theorist's point of view. In this connection, I recall that a recent measurement of the neutron lifetime [31] suggests a substantially bigger $\left|V_{u d}\right|$, which together with CKM unitarity would imply a value for $\left|V_{u s}\right|$ even smaller than the $\tau$ decay result (3.2). However, the neutron lifetime from Ref. [31] is incompatible with the present world average [32]. 


\section{$3.2 P_{l 2}$ decays $(P=\pi, K)$}

Because of the $V-A$ structure of charged currents the ratios $R_{e / \mu}^{(P)}=\Gamma\left(P \rightarrow e v_{e}[\gamma]\right) / \Gamma\left(P \rightarrow \mu v_{\mu}[\gamma]\right)$ are helicity suppressed. In order to serve as sensitive probes for new physics, the Standard Model values for these ratios must be known as precisely as possible. The current experimental values are [32, 24,

$$
R_{e / \mu}^{(\pi)}=1.230(4) \cdot 10^{-4}, \quad R_{e / \mu}^{(K)}=2.457(32) \cdot 10^{-5} .
$$

Turning off electromagnetic corrections, the ratios are given by the classic values

$$
R_{e / \mu}^{P}=\frac{m_{e}^{2}}{m_{\mu}^{2}}\left(\frac{M_{P}^{2}-m_{e}^{2}}{M_{P}^{2}-m_{\mu}^{2}}\right)^{2}
$$

to all orders in the chiral expansion. Nontrivial structure dependent effects appear only for $e \neq 0$.

The corrections of $O\left(e^{2} p^{2}\right)$ correspond to a point-like approximation. The first systematic calculation to $O\left(e^{2} p^{4}\right)$, sensitive to the meson structure (form factors), was recently performed by Cirigliano and Rosell [33]. The setup is the Lagrangian of Table 1 including photons and leptons. In fact, the 2-loop calculation requires the determination of a LEC of the Lagrangian of $O\left(e^{2} p^{4}\right)$ that is not included in Table 1 because the complete Lagrangian is not yet available. The relevant LEC is obtained by matching the relevant form factors with large- $N_{c}$ QCD [33]. The associated uncertainty is accounted for in the final error estimate. In contrast, the chiral double logs are model independent.

\begin{tabular}{|l||c|c|c|}
\hline & Cirigliano, Rosell [33] & Marciano, Sirlin [34] & Finkemeier [35] \\
\hline$R_{e / \mu}^{(\pi)} \cdot 10^{4}$ & $1.2352 \pm 0.0001$ & $1.2352 \pm 0.0005$ & $1.2354 \pm 0.0002$ \\
$R_{e / \mu}^{(K)} \cdot 10^{5}$ & $2.477 \pm 0.001$ & & $2.472 \pm 0.001$ \\
\hline
\end{tabular}

Table 2: Theoretical predictions for $R_{e / \mu}^{(P)}$.

Including photon emission and summing up the leading $\operatorname{logs} \alpha^{n} \log ^{n}\left(m_{\mu} / m_{e}\right)$ [34], the final results are displayed in Table 2 and compared with previous calculations. For the pionic ratio, the previous predictions are confirmed with better precision. The discrepancy in the predictions for the kaonic ratio is mainly due to the fact that the asymptotic behaviour of form factors in the model used in Ref. [35] is incompatible with QCD. Comparing the theoretical with the present experimental values in (3.3), there is room for new physics to be detected with more accurate measurements.

\section{Nonleptonic $K$ decays}

CHPT has also had a big impact on nonleptonic $K$ decays. However, in contrast to semileptonic decays, already at NLO, $O\left(G_{F} p^{4}\right)$, not all LECs are known. Therefore, nonleptonic decays without any LECs at NLO have always been theorists' favourites. They are unambiguously predicted to $O\left(G_{F} p^{4}\right)$ in terms of the two known LECs of lowest order. Clearly, estimates of NNLO contributions are needed for a meaningful comparison with experiment. 
Two early examples are the decays $K_{S} \rightarrow \gamma \gamma$ [36] and $K_{L} \rightarrow \pi^{0} \gamma \gamma$ [37] where recent experimental developments have greatly clarified the situation. Estimates of higher-order corrections were made in two directions.

- Rescattering corrections can be calculated in a largely model independent way from unitarity [38-40]. For $K_{S} \rightarrow \gamma \gamma$ these corrections can essentially be expressed in terms of the rates for $K \rightarrow \pi \pi$. On the other hand, the corrections are more involved for $K_{L} \rightarrow \pi^{0} \gamma \gamma$ and they are sizable.

- A comprehensive treatment of LECs of $O\left(G_{F} p^{6}\right)$ is beyond present technology. From experience with strong amplitudes, one expects vector meson exchange to be important whenever vector mesons contribute at all. They cannot contribute to $K_{S} \rightarrow \gamma \gamma$ but they could have a substantial influence on $K_{L} \rightarrow \pi^{0} \gamma \gamma$. Assuming that vector meson exchange is indeed dominating at $O\left(G_{F} p^{6}\right)$, the contributing LECs of $O\left(G_{F} p^{6}\right)$ can be parametrized by a single dimensionless constant $a_{V}$ [39, 41].

\begin{tabular}{cc}
\hline \hline & $B\left(K_{S} \rightarrow \gamma \gamma\right) \cdot 10^{6}$ \\
\hline KLOE [42] & $2.26(12)(06)$ \\
CHPT [36] & $2.15(20)$ \\
\hline \hline
\end{tabular}

Figure 3: Comparison of experimental results with the CHPT prediction for $K_{S} \rightarrow \gamma \gamma$ (courtesy of Matteo Martini).

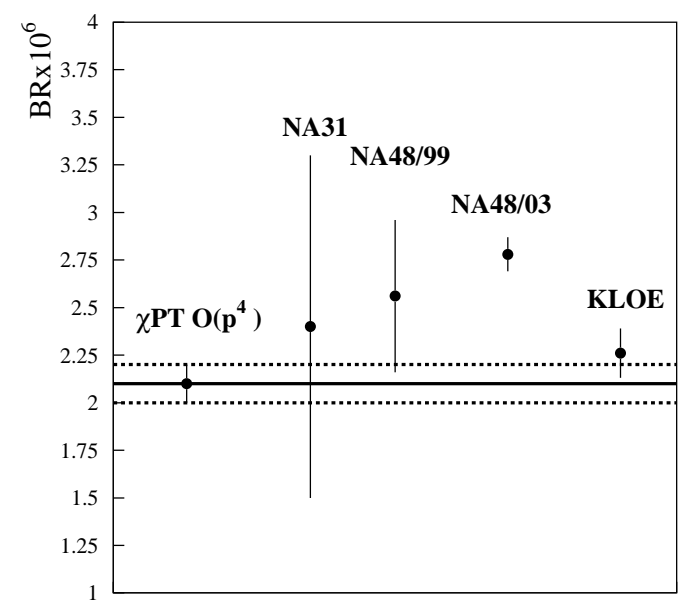

Updating the original calculation [36] and assigning an uncertainty of $10 \%$ to the rate for $K_{S} \rightarrow \gamma \gamma$ in view of the above discussion, the CHPT prediction for the branching ratio is

$$
\left.B\left(K_{S} \rightarrow \gamma \gamma\right)\right|_{\mathrm{CHPT}}=(2.15 \pm 0.20) \cdot 10^{-6} .
$$

As displayed in Fig. B, the first experimental results were consistent with expectations within large errors but the last precise measurement of NA48 [43] was significantly bigger than the CHPT branching ratio (4.1). To my knowledge, no serious attempt was made to find a mechanism that would raise the chiral prediction (4.1) substantially. The most recent precise measurement from KLOE [42] shown in Fig. 3 is therefore most welcome, reestablishing the agreement between theory and experiment.

Turning to $K_{L} \rightarrow \pi^{0} \gamma \gamma$, experimental data have always been in agreement with the chiral prediction that the pion-loop contribution dominates the two-photon spectrum. However, until very recently there were conflicting experimental results for the decay rate that is affected significantly 
by both rescattering corrections and by higher-order LECs. With the final analysis of $\mathrm{KTeV}$ for $K_{L} \rightarrow \pi^{0} \gamma \gamma$ released this year [44, there is now excellent agreement between theory and experiment for both rate and spectrum in terms of the single parameter $a_{V}$. The final results

$$
\begin{aligned}
B\left(K_{L} \rightarrow \pi^{0} \gamma \gamma\right) \cdot 10^{6} & = \begin{cases}1.36 \pm 0.03 \pm 0.03 \pm 0.03 & \mathrm{NA} 48 \text { 445 } \\
1.29 \pm 0.03 \pm 0.05 & \mathrm{KTeV}\end{cases} \\
a_{V} & =\left\{\begin{array}{ll}
-0.46 \pm 0.03 \pm 0.04 & \mathrm{NA48} \\
-0.31 \pm 0.05 \pm 0.07 & \mathrm{KTeV}
\end{array}\right]
\end{aligned}
$$

document that patience is sometimes appropriate for nonleptonic $K$ decays. An important consequence is that the $\mathrm{CP}$ conserving contribution to $K_{L} \rightarrow \pi^{0} e^{+} e^{-}$via $K_{L} \rightarrow \pi^{0} \gamma^{*} \gamma^{*} \rightarrow \pi^{0} e^{+} e^{-}$is definitely negligible compared with the $\mathrm{CP}$ violating amplitudes.

It would be premature to conclude that higher-order corrections in nonleptonic $K$ decays are under control in general but the situation for $K_{S} \rightarrow \gamma \gamma$ and $K_{L} \rightarrow \pi^{0} \gamma \gamma$ is certainly encouraging. More experimental results are already available or forthcoming. A recent review of nonleptonic $K$ decays in CHPT can be found in Ref. [46].

\section{Other topics}

Finally, I briefly review here a few other interesting recent developments in CHPT.

\subsection{Radiative pion decay $\pi \rightarrow e v_{e} \gamma$}

Resonance contributions to the vector and axial-vector form factors governing the structure dependent part of the $\pi \rightarrow e v_{e} \gamma$ amplitude were calculated in Ref. [47]. The relevant LECs of $O\left(p^{6}\right)$ were also estimated in Ref. [48] where in addition radiative corrections for the process were performed in a CHPT framework. The very recent PIBETA experiment [49] finds no evidence for a previously reported tensor contribution and is in agreement with theoretical expectations.

\subsection{Chiral SU(2) vs. $\mathrm{SU}(3)$}

In the limit where the strange quark mass $m_{s}$ is much bigger than $m_{u}, m_{d}$ and all external momenta, chiral $S U(3)$ reduces to the two-flavour case. Such a procedure allows to determine the $m_{s}$-dependence of LECs in chiral $S U(2)$ and it provides relations between the LECs of the twoand three-flavour chiral Lagrangians. To $O\left(p^{4}\right)$, such relations were established already in the classic paper of Gasser and Leutwyler [3]. Recently, the relations have been worked out to $O\left(p^{6}\right)$ [50]. The results are expected to be useful for determining some of the LECs of $O\left(p^{6}\right)$ to allow for an efficient comparison between theory and experiment to two-loop accuracy.

\section{$5.3 \eta \rightarrow 3 \pi$ decays}

The decays $\eta \rightarrow 3 \pi$ are prominent examples for large chiral corrections. A complete $O\left(p^{6}\right)$ calculation was performed recently by Bijnens and Ghorbani [51]. The corrections of $O\left(p^{6}\right)$ turn out to be somewhat larger than previously obtained with dispersive methods [52]. However, a very recent measurement of both $\eta \rightarrow 3 \pi^{0}$ and $\eta \rightarrow \pi^{+} \pi^{-} \pi^{0}$ by KLOE [53] indicates that there are still discrepancies between theory and experiment, especially in the slope parameters. Once again, it may be necessary to have better theoretical control of the $O\left(p^{6}\right)$ LECs involved. 


\section{Conclusions}

Thirty years after its conception, there is still significant progress in CHPT along several lines.

The impressive precision in pion pion scattering obtained by combining CHPT with dispersion theory is now being challenged experimentally, with data mainly from $K_{e 4}$ and $K \rightarrow 3 \pi$ decays.

Kaon physics is a traditional stronghold of CHPT. Even if some issues remain to be clarified, $K_{l 3}$ decays provide at present the best source for extracting the CKM matrix element $V_{u s}$. The recent calculation of the ratios $\Gamma\left(P \rightarrow e v_{e}\right) / \Gamma\left(P \rightarrow \mu v_{\mu}\right)$ to $O\left(e^{2} p^{4}\right)$ with very small theoretical uncertainties constitute a challenge for experimental confirmation or the possible detection of new physics. The history of the nonleptonic decays $K_{S} \rightarrow \gamma \gamma$ and $K_{L} \rightarrow \pi^{0} \gamma \gamma$ suggests that sometimes patience is called for in this field.

In general, CHPT stands for precision physics at low energies in several areas, allowing for significant tests of the Standard Model. In particular, CHPT has established itself as the only reliable method for isospin violating and electromagnetic corrections. Further progress in the field will depend on progress in the determination of LECs.

\section{References}

[1] S. Weinberg, Physica A 96 (1979) 327.

[2] J. Gasser and H. Leutwyler, Annals Phys. 158 (1984) 142.

[3] J. Gasser and H. Leutwyler, Nucl. Phys. B 250 (1985) 465.

[4] U.G. Meißner, these Proceedings.

[5] S. Necco, these Proceedings.

[6] H. Leutwyler, these Proceedings.

[7] A. Pich, these Proceedings.

[8] B. Ananthanarayan et al., Phys. Rept. 353 (2001) 207 [arXiv:hep-ph/0005297].

[9] J. Bijnens et al., Phys. Lett. B 374 (1996) 210 [arXiv:hep-ph/9511397]; Nucl. Phys. B 508 (1997) 263 [Erratum-ibid. B 517 (1998) 639] [arXiv:hep-ph/9707291].

[10] G. Colangelo, J. Gasser and H. Leutwyler, Nucl. Phys. B 603 (2001) 125 [arXiv:hep-ph/0103088].

[11] G. Colangelo, J. Gasser and A. Rusetsky, arXiv:0811.0775 [hep-ph].

[12] J. R. Batley et al. [NA48/2 Collaboration], Eur. Phys. J. C 54 (2008) 411.

[13] S. Pislak et al. [E865 Collaboration], Phys. Rev. D 67 (2003) 072004 [arXiv:hep-ex/0301040].

[14] B. Bloch-Devaux, these Proceedings.

[15] J. R. Batley et al. [NA48/2 Collaboration], Phys. Lett. B 633 (2006) 173 [arXiv:hep-ex/0511056].

[16] N. Cabibbo, Phys. Rev. Lett. 93 (2004) 121801 [arXiv:hep-ph/0405001].

[17] U. G. Meißner, G. Müller and S. Steininger, Phys. Lett. B 406 (1997) 154 [Erratum-ibid. B 407 (1997) 454] [arXiv:hep-ph/9704377].

[18] N. Cabibbo and G. Isidori, JHEP 0503 (2005) 021 [arXiv:hep-ph/0502130].

[19] E. Gamiz, J. Prades and I. Scimemi, Eur. Phys. J. C 50 (2007) 405 [arXiv:hep-ph/0602023]. 
[20] K. Kampf et al., arXiv:0810.1906 [hep-ph] and in preparation.

[21] G. Colangelo et al., Phys. Lett. B 638 (2006) 187 [arXiv:hep-ph/0604084].

[22] M. Bissegger et al., Nucl. Phys. B 806 (2009) 178 [arXiv:0807.0515 [hep-ph]].

[23] J. Bijnens et al., in The Second DAPHNE Physics Handbook (1995), p.315; L. Maiani, G. Pancheri and N. Paver, eds. [arXiv:hep-ph/9411311].

[24] M. Antonelli et al. [FlaviaNet Working Group on Kaon Decays], arXiv:0801.1817 [hep-ph].

[25] V. Cirigliano, M. Giannotti and H. Neufeld, JHEP 0811 (2008) 006 [arXiv:0807.4507 [hep-ph]].

[26] P. A. Boyle et al., Phys. Rev. Lett. 100 (2008) 141601 [arXiv:0710.5136 [hep-lat]].

[27] E. Gamiz et al., PoS KAON (2008) 008 [arXiv:0709.0282 [hep-ph]].

[28] K. Maltman et al., Int. J. Mod. Phys. A 23 (2008) 3191 [arXiv:0807.3195 [hep-ph]].

[29] T. Eronen et al., Phys. Rev. Lett. 100 (2008) 132502 [Erratum-ibid. 100 (2008) 149902] [arXiv:0712.3463 [nucl-ex]].

[30] V. Cirigliano et al., Eur. Phys. J. C 27 (2003) 255 [arXiv:hep-ph/0209226].

[31] A. Serebrov et al., Phys. Lett. B 605 (2005) 72 [arXiv:nucl-ex/0408009].

[32] C. Amsler et al. (Particle Data Group), Physics Letters B 667 (2008) 1.

[33] V. Cirigliano and I. Rosell, Phys. Rev. Lett. 99 (2007) 231801 [arXiv:0707.3439 [hep-ph]]; JHEP 0710 (2007) 005 [arXiv:0707.4464 [hep-ph]].

[34] W. J. Marciano and A. Sirlin, Phys. Rev. Lett. 71 (1993) 3629.

[35] M. Finkemeier, Phys. Lett. B 387 (1996) 391 [arXiv:hep-ph/9505434].

[36] G. D’Ambrosio and D. Espriu, Phys. Lett. B 175 (1986) 237;

J. L. Goity, Z. Phys. C 34 (1987) 341.

[37] G. Ecker, A. Pich and E. de Rafael, Phys. Lett. B 189 (1987) 363;

L. Cappiello and G. D'Ambrosio, Nuovo Cim. A 99 (1988) 155.

[38] L. Cappiello, G. D’Ambrosio and M. Miragliuolo, Phys. Lett. B 298 (1993) 423.

[39] A. G. Cohen, G. Ecker and A. Pich, Phys. Lett. B 304 (1993) 347.

[40] J. Kambor and B. R. Holstein, Phys. Rev. D 49 (1994) 2346 [arXiv:hep-ph/9310324].

[41] G. D’Ambrosio and J. Portolés, Nucl. Phys. B 492 (1997) 417 [arXiv:hep-ph/9610244].

[42] F. Ambrosino et al. [KLOE Collaboration], JHEP 0805 (2008) 051 [arXiv:0712.1744 [hep-ex]].

[43] A. Lai et al. [NA48 Collaboration], Phys. Lett. B 551 (2003) 7 [arXiv:hep-ex/0210053].

[44] E. Abouzaid et al. [KTeV Collaboration], Phys. Rev. D 77 (2008) 112004 [arXiv:0805.0031 [hep-ex]].

[45] A. Lai et al. [NA48 Collaboration], Phys. Lett. B 536 (2002) 229 [arXiv:hep-ex/0205010].

[46] J. Prades, PoS KAON (2008) 022 [arXiv:0707.1789 [hep-ph]].

[47] V. Mateu and J. Portolés, Eur. Phys. J. C 52 (2007) 325 [arXiv:0706.1039 [hep-ph]].

[48] R. Unterdorfer and H. Pichl, Eur. Phys. J. C 55 (2008) 273 [arXiv:0801.2482 [hep-ph]].

[49] M. Bychkov et al. [PIBETA Collaboration], arXiv:0804.1815 [hep-ex]. 
[50] J. Gasser et al., Phys. Lett. B 652 (2007) 21 [arXiv:0706.0955 [hep-ph]] and in preparation.

[51] J. Bijnens and K. Ghorbani, JHEP 0711 (2007) 030 [arXiv:0709.0230 [hep-ph]].

[52] J. Kambor, C. Wiesendanger and D. Wyler, Nucl. Phys. B 465 (1996) 215 [arXiv:hep-ph/9509374];

A. V. Anisovich and H. Leutwyler, Phys. Lett. B 375 (1996) 335 [arXiv:hep-ph/9601237].

[53] F. Ambrosino et al. [KLOE Collaboration], JHEP 0805 (2008) 006 [arXiv:0801.2642 [hep-ex]]. 